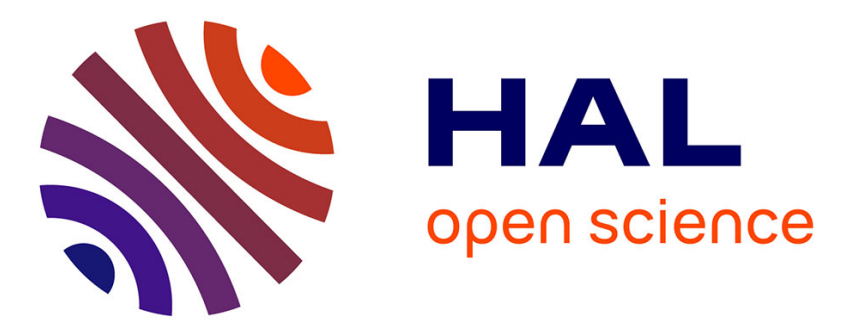

\title{
Réalisation d'un circuit de discrimination neutron-gamma
}

\author{
A. Adam, J. Cabe, M. Cance, M. Laurat, J.C. Ponce
}

\section{To cite this version:}

A. Adam, J. Cabe, M. Cance, M. Laurat, J.C. Ponce. Réalisation d'un circuit de discrimination neutron-gamma. Revue de Physique Appliquée, 1969, 4 (2), pp.262-264. 10.1051/rphysap:0196900402026201 . jpa-00243252

\section{HAL Id: jpa-00243252 https://hal.science/jpa-00243252}

Submitted on 1 Jan 1969

HAL is a multi-disciplinary open access archive for the deposit and dissemination of scientific research documents, whether they are published or not. The documents may come from teaching and research institutions in France or abroad, or from public or private research centers.
L'archive ouverte pluridisciplinaire HAL, est destinée au dépôt et à la diffusion de documents scientifiques de niveau recherche, publiés ou non, émanant des établissements d'enseignement et de recherche français ou étrangers, des laboratoires publics ou privés. 


\title{
RÉALISATION D’UN GIRGUIT DE DISGRIMINATION NEUTRON-GAMMA
}

\author{
A. ADAM, J. CABE, M. CANCE, M. LAURAT et J. C. PONCE, \\ Centre d’Études de Bruyères-le-Châtel.
}

\begin{abstract}
Résumé. - Nous présentons une discrimination neutron-gamma dont le principe est' dû à Roush, qui permet une bonne séparation entre les électrons et les protons de recul d'une énergie supérieure à $150 \mathrm{keV}$. Ce circuit, d'une très bonne efficacité, libère l'anode du photomultiplicateur pour les mesures de temps-de-vol.
\end{abstract}

Abstract. - We present a neutron-gamma discrimination circuit of the Roush principle which allows good separation between electrons and recoil protons of energy above $150 \mathrm{keV}$. This circuit has a very good efficiency. It leaves the anode of the photomultiplier free for time-of-flight measurements.

I. Introduction. - La discrimination des neutrons et des gamma détectés par un scintillateur organique associé à un photomultiplicateur a fait l'objet de nombreuses études [1-4]. Nous avons réalisé un circuit de discrimination sur le principe exposé par Roush [3]. L'intérêt de ce montage est de permettre une séparation entre les deux rayonnements excellente et de même qualité pour des énergies de neutrons comprises entre 0,4 et $14 \mathrm{MeV}$.
II. Schéma de principe. - Les impulsions lumineuses correspondant aux protons de recul dus aux neutrons ou aux électrons excités par les gamma présentent des temps de décroissance différents; l'idée de Roush [3], que nous avons reprise, était de différentier le signal d'une dynode et de convertir en amplitude la différence des temps de passage à zéro des impulsions différentiées. Le circuit réalisé ( fig. 1) a été mis au point dans le cas d'un scintillateur NE 213 couplé à un PM 56 AVP. 


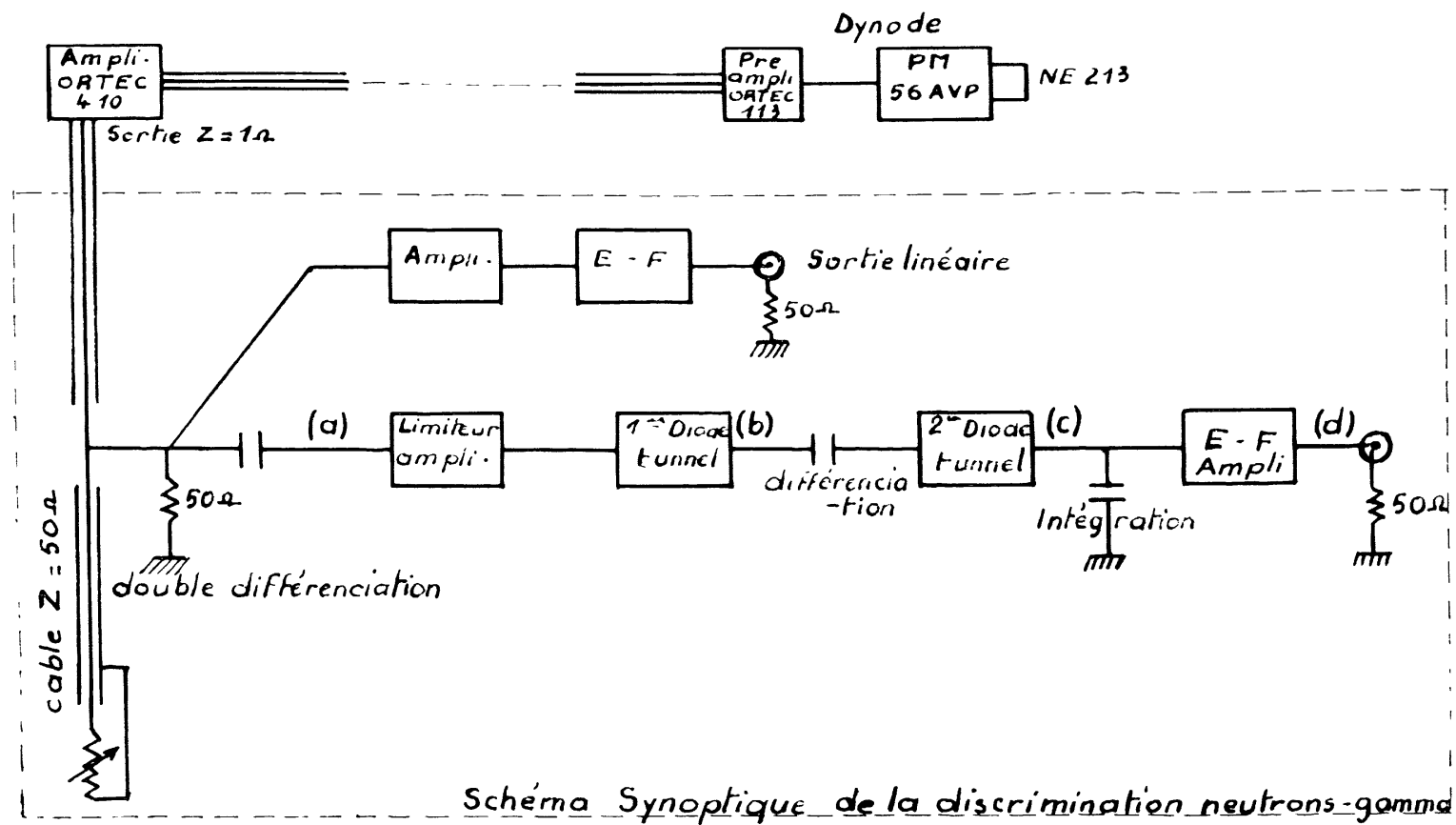
FIG. 1.
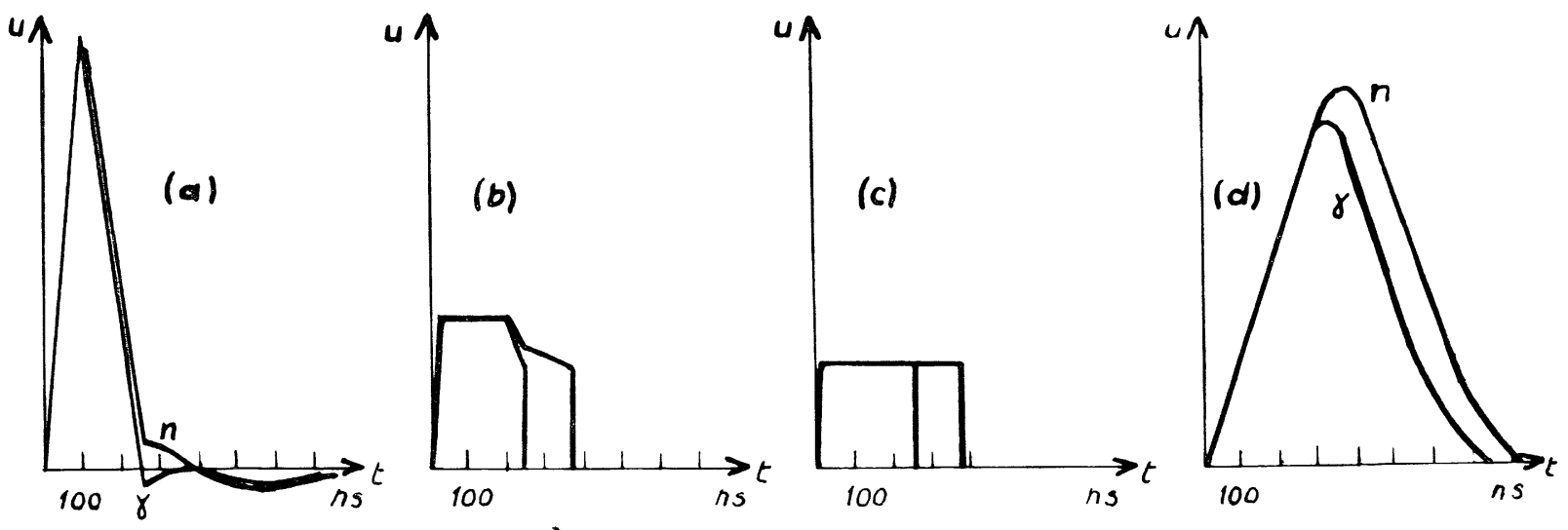

FIG. 2.

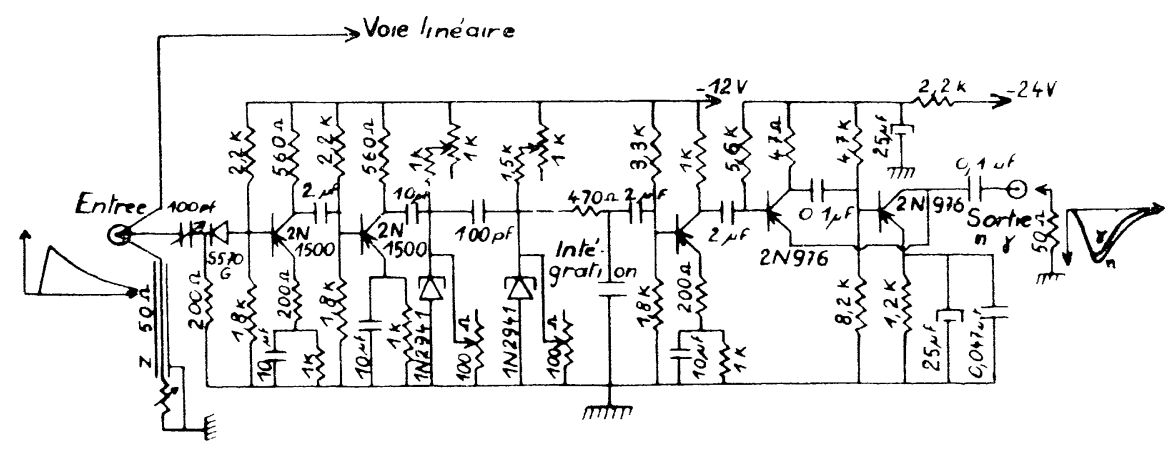

FIG. 3. 


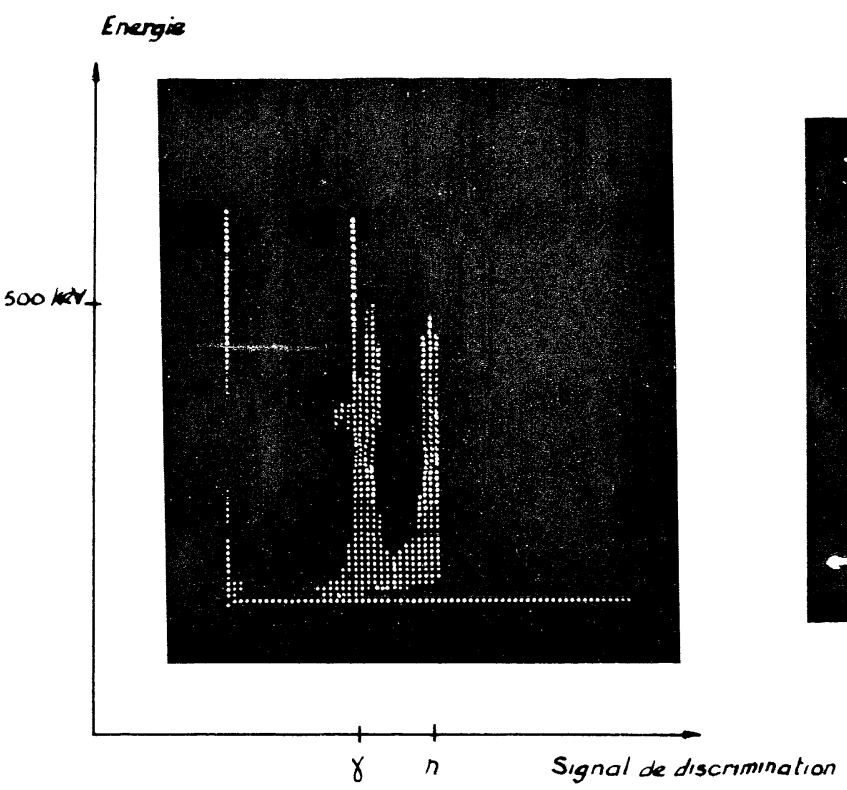

(a)

Le signal d'une dynode est intégré par un préamplificateur et un amplificateur avec une constante de temps (100 ns environ) comprise entre les deux temps de décroissance, lent et rapide. Le signal délivré par la sortie très basses impédances $(1 \Omega)$ de l'amplificateur est différentié à l'aide d'un câble fermé sur sa résistance critique et d'une capacité en série à l'entrée du circuit de discrimination. Le temps de passage à zéro des impulsions correspondant aux protons ou aux électrons est alors différent ( fig. 2 a), la dynamique d'entrée de cet étage est assez grande grâce à son montage en limiteur amplificateur. Le deuxième étage comprend une première diode tunnel qui conduit entre le front de montée et le passage à zéro de l'impulsion : ainsi la largeur de l'impulsion aux bornes de cette diode est proportionnelle au temps du «zéro crossing » ( fig. $2 \mathrm{~b}$ ). Après une différentiation par capacité, une seconde diode tunnel montée en bistable permet d'obtenir le signal indiqué ( $f g .2 \mathrm{c}$ ). Une simple intégration permet alors la séparation en deux niveaux d'amplitudes différentes, mieux définis que si l'on intégrait directement l'impulsion délivrée par la première diode tunnel ( fig. $2 \mathrm{~d}$ ). Le schéma détaillé du circuit de discrimination est représenté figure 3 .

III. Résultats expérimentaux. - Nous avons utilisé notre montage pour diverses expériences de neutronique (détermination de sections efficaces totales et différentielles), soit à basse énergie (de 0,3 à $1,2 \mathrm{MeV}$ ), soit à $14 \mathrm{MeV}$. Cette souplesse d'utilisation est possible grâce à une dynamique d'entrée assez grande : les impulsions à l'entrée du discriminateur peuvent avoir un niveau compris entre $50 \mathrm{mV}$ et $8 \mathrm{~V}$ (dynamique en tension de 160). La figure 4 a montre une représentation biparamétrique des impulsions en fonction de leur amplitude linéaire à la sortie du photomultiplicateur; l'énergie des neutrons était de $500 \mathrm{keV}$. Cette figure montre que la séparation $n-\gamma$ est très bonne au-dessus de $150 \mathrm{keV}$. La figure $4 \mathrm{~b}$ montre trois spectres obtenus avec des neutrons de $14 \mathrm{MeV}$. Ces trois spectres de la voie linéaire représentent, de haut en bas : le spectre sans discrimination, le spectre en coïncidence avec le pic « neutrons » de la discrimination et le spectre en coïncidence avec le pic « gamma » de la discrimination.

IV. Conclusion. - Ce système présente plusieurs avantages sur les systèmes du type Brooks ou Owen : 1) bonne séparation $n-\gamma$, indépendante de l'énergie de ces particules au-delà de $150 \mathrm{keV}$; 2) utilisation d'une seule dynode pour la discrimination, l'anode restant disponible pour les informations en temps; 3) la largeur de l'impulsion de sortie, réglée par l'intégration du signal, est de $1 \mu$ s à mi-hauteur, ce qui autorise des taux de comptage assez élevés; 4) bonne stabilité : pendant une utilisation d'un mois, la dérive était de l'ordre de quelques pour cent. Cependant, le réglage optimal de la double différentiation est assez délicat à obtenir.

\section{BIBLIOGRAPHIE}

[1] Brooks (F. D.), Nucl. Instr. Methods, 1959, 4, 157.

[2] Ovwen (R. B.), I.R.E. Trans. Nucl. Sci., 1961, NS 8, 285
[3] Roush (M. L.) et al., Nucl. Instr. Methods, 1964, 31, 112.

[4] SCHWEIMER (W.), Nucl. Instr. Methods, 1966, 39, 343.

[5] VARGA, Nucl. Instr. Methods, 1961, 14, 24. 